\title{
Acute Myeloid Leukemia with NUP98-RARG Gene Fusion Similar to Acute Promyelocytic Leukemia: Case Report and Literature Review
}

This article was published in the following Dove Press journal: OncoTargets and Therapy

\author{
Shandong Tao ${ }^{1,2, *}$ \\ Lixiao Song ${ }^{1,2, *}$ \\ Yuan Deng ${ }^{1,2}$ \\ Yue Chen ${ }^{1,2}$ \\ Yuye Shi ${ }^{1,2}$ \\ Yimin Gan ${ }^{1,2}$ \\ Zhikui Deng ${ }^{1,2}$ \\ Banghe Ding ${ }^{1,2}$ \\ Zhengmei $\mathrm{He}^{1,2}$ \\ Chunling Wang ${ }^{1,2}$ \\ Liang $\mathrm{Yu}^{1,2}$
}

'Department of Hematology, The Affiliated Huai'an No.I People's Hospital of Nanjing Medical University, Huai'an, Jiangsu 223300, People's Republic of China; ${ }^{2}$ Key Laboratory of Hematology of Nanjing Medical University, Nanjing 210029, People's Republic of China

*These authors contributed equally to this work
Correspondence: Liang Yu; Chunling Wang

I Huang River Road West, Huai'an, jiangsu 223300, People's Republic of China

$\mathrm{Tel} / \mathrm{Fax}+86-51780872603$

Email yuliangha@163.com;

wcl6506@I63.com

\begin{abstract}
Retinoic acid receptor gamma (RARG) belongs to the nuclear receptor superfamily and has $90 \%$ homology to RAR alpha (RARA) and RAR beta. The promyelocytic leukemia $(P M L)-R A R A$ fusion gene has been implicated in acute promyelocytic leukemia (APL). RARG gene rearrangement has been identified in a rare subtype of acute myeloid leukemia (AML) that resembles APL. To date, only 10 cases of gene rearrangements involving RARG (nucleoporin [NUP]98-RARG, promyelocytic leukemia protein-RARG, cleavage and polyadenylation-specific factor 6-RARG, or nucleophosmin [NPM]1-RARGNPM1) have been reported. These patients show characteristics similar to APL, including bone marrow morphology, coagulation abnormality, and immunophenotype; however, they are resistant to all-trans retinoic acid and arsenic trioxide treatment. Moreover, there is no optimal therapeutic regimen for this subtype of AML. In this study, we report the clinical presentation and experimental findings of a case of AML with NUP98-RARG gene fusion similar to APL and review other cases of $R A R G$ gene rearrangement described in the literature.
\end{abstract}

Keywords: acute myeloid leukemia, acute promyelocytic leukemia, NUP98-RARG, RARG rearrangement

\section{Introduction}

Acute myeloid leukemia (AML) is a highly heterogeneous and malignant clonal disease of hemopoietic stem cells characterized by uncontrolled proliferation and blocked differentiation of myeloid lineage blasts. ${ }^{1}$ Acute promyelocytic leukemia (APL) is a specific type of AML, accounting for 5-15\% of total AML cases. ${ }^{2}$ The fusion gene promyelocytic leukemia protein $(P M L)$-retinoic acid receptor alpha $(R A R A)$, which is generated by the chromosomal translocation $\mathrm{t}(15 ; 17)(\mathrm{q} 22 ; \mathrm{q} 21)$, is implicated in the pathogenesis of APL. All-trans retinoic acid (ATRA) and arsenic trioxide (ATO) have been shown to induce myeloid blast differentiation in APL; ${ }^{3}$ and it is recommended that patients with APL who have relapsed after ATRA plus chemotherapy be treated with a combined ATRA plus ATO-based regimen. ${ }^{4}$

RAR gamma (RARG) plays a key role in maintaining the self-renewal and differentiation of hematopoietic stem cells. RARG gene rearrangements have been detected in AML as a rare specific subtype with a clinical presentation resembling that of APL $;^{5}$ however, these patients do not respond to treatment with ATRA and ATO. As such, the role of $R A R G$ in AML remains unclear. 
The first AML patient with a nucleoporin (NUP)98$R A R G$ gene rearrangement was identified in $2011 .{ }^{6}$ Since then, 9 additional cases of $R A R G$ rearrangement have been reported, including 2 with $N U P 98-R A R G$ fusions. ${ }^{7-13}$ Here we describe a case of NUP98-RARG gene fusion in an AML patient with the classic morphologic features and immunophenotype of APL.

\section{Materials and Methods Case Presentation}

This study was approved by the institutional review board of The Affiliated Huai'an No.1 People's Hospital of Nanjing Medical University. On April 27, 2018, a 47year-old female patient was admitted to our hospital presenting with skin ecchymosis and vaginal bleeding. The peripheral blood count revealed a white blood cell (WBC) count of $7.91 \times 10^{9} / 1$, hemoglobin $(\mathrm{Hg})$ level of $84 \mathrm{~g} / \mathrm{l}$, and platelet count of $14 \times 10^{9} / 1$. Prothrombin time was 16.9 s (ref. 9.0-13.0 s), activated partial thromboplastin time was $40.7 \mathrm{~s}$ (ref. $20.0-40.0 \mathrm{~s}$ ), fibrinogen level was $1.66 \mathrm{~g} / 1$ (ref. $2.00-4.00 \mathrm{~g} / \mathrm{l}$ ), and D-dimer level was $20 \mu \mathrm{g} / \mathrm{mL}$ (ref. $0-0.50 \mu \mathrm{g} / \mathrm{mL}$ ). A bone marrow (BM) smear showed hypercellularity, with $96.5 \%$ abnormal promyelocytic granulocytes. Auer bodies were observed in some cells. The smear was strongly positive for peroxidase staining (Figure 1A-C). An immunophenotype analysis showed positivity for myeloperoxidase, cluster of differentiation (CD)13, CD33, human leukocyte antigen-DR, and CD56 (Figure 2). The results of second-generation gene sequencing and reverse transcription-polymerase chain reaction (RT-PCR) were indeterminate. The initial diagnosis was APL. The patient was treated with ATRA (20 mg twice a day) and ATO (10 mg per day). After 14 days of treatment, the patient experienced chest tightness, dyspnea, systemic edema, and pleural effusion, consistent with differentiation syndrome. A chest computed tomography scan and color Doppler ultrasound were performed (Figure 3). WBC count did not increase with treatment and the coagulation abnormality did not show significant improvement: WBC count was $9.4 \times 10^{9} / \mathrm{l}, \mathrm{Hg}$ level was $59 \mathrm{~g} / \mathrm{l}$, and platelet count was $38 \times 10^{9} / 1$. Prothrombin time was $18.3 \mathrm{~s}$, fibrinogen level was $0.91 \mathrm{~g} / \mathrm{l}$, and D-dimer level was $20 \mu \mathrm{g} / \mathrm{mL}$. The karyotype was $45, \mathrm{X},-\mathrm{X}, \operatorname{del}(9)$ (q13q22), t(11;12)(p15;q13) in 20/20 analyzed metaphases
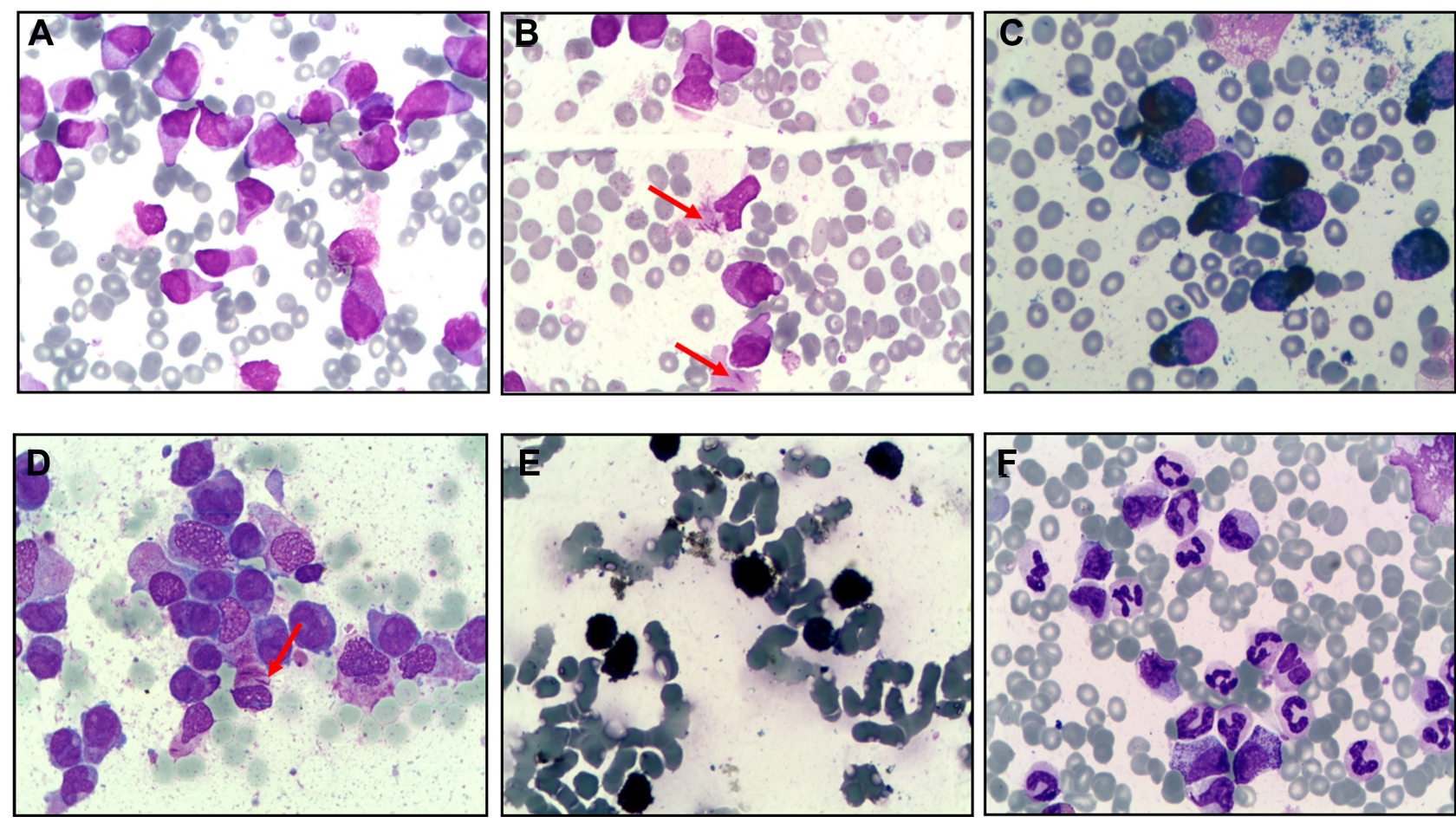

Figure I Morphologic features of an AML patient with NUP98-RARG gene fusion. (A, B, D) Promyelocytes with hypergranulated cytoplasm, invaginated nuclei, and Auer rods are indicated with red arrows (Wright-Giemsa stained BM smear, 1000x magnification). (C, E) BM smear strongly positive for peroxidase staining. Morphologic features were observed at the newly diagnosed stage (A-C) and 20 days after treatment with ATRA and ATO (D, E). The BM smear showed $95.5 \%$ abnormal promyelocytic granulocytes, with an occasional Auer body in the form of an Auer bundle; the peroxidase staining positive rate was $100 \%$, indicating that there was no remission. (F) BM smear revealing complete remission. 
A

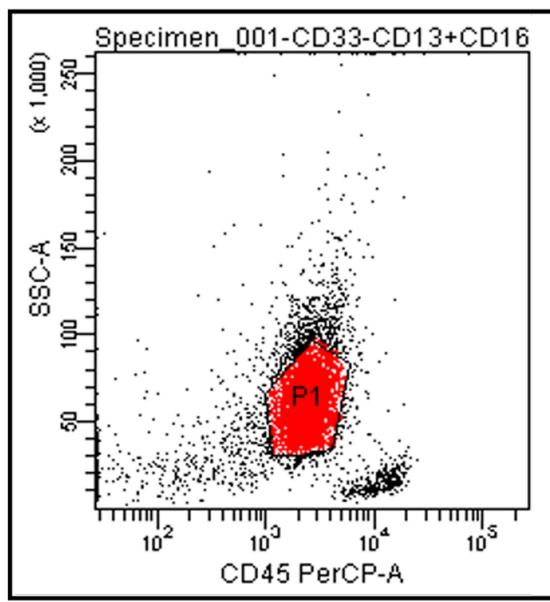

D

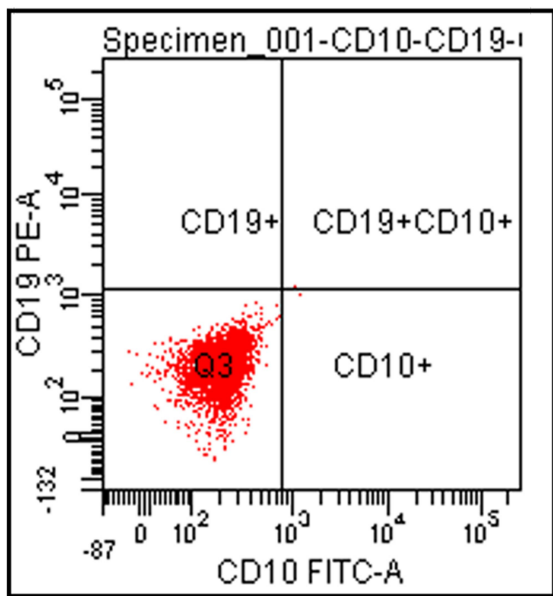

B

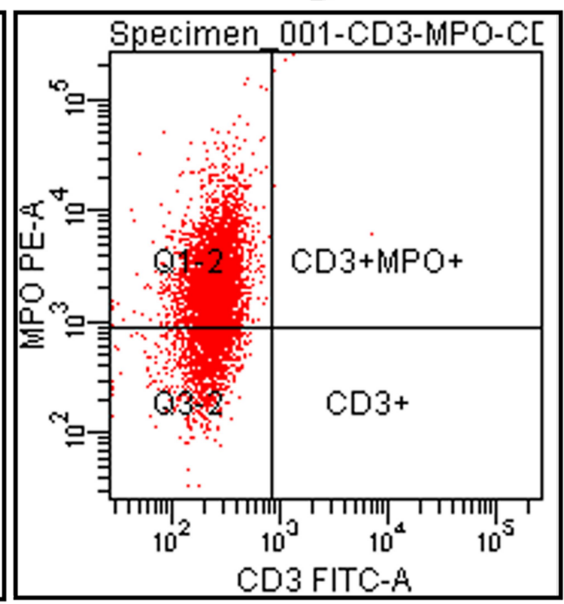

E

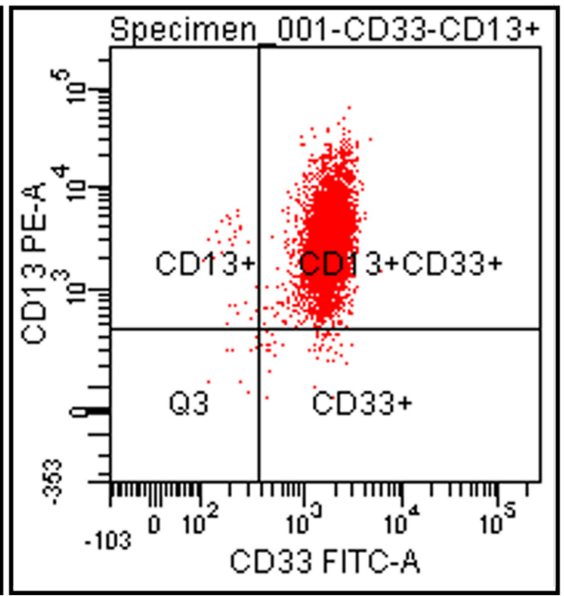

C

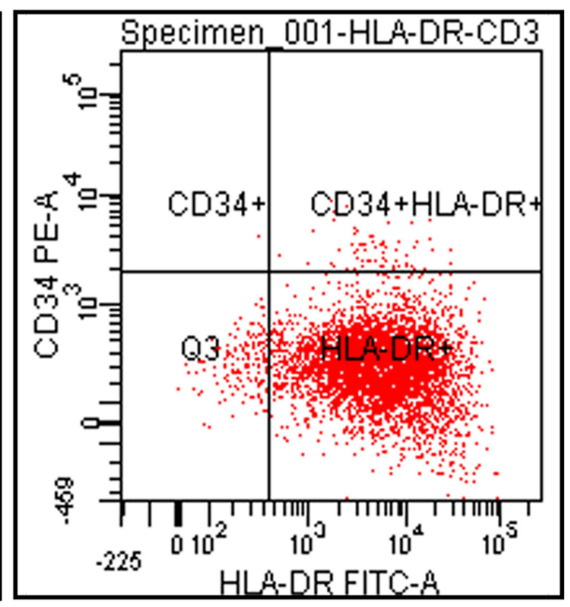

Figure 2 Flow cytometry scatterplots showing an immunotype consisting of abnormal populations in an AML patient with NUP98-RARG gene fusion. (A) CD45 gating; a group of abnormal cells is circled. (B) Positive myeloperoxidase (MPO) and negative CD3 expression. (C) Positive human leukocyte antigen (HLA)-DR and negative CD34 expression. (D) CDI9 and CDI0 negativity. (E) CDI3 and CD33 positivity. (F) CD56 and CDI6 negativity.

(Figure 4); fluorescence quantitative PCR detection of the $P M L-R A R A$ fusion transcript was negative, but mutations were detected in several genes at various frequencies including isocitrate dehydrogenase (IDH)2 (37.68\%), teneleven translocation (TET)2 (49.72\%), additional sex combs-like $(A S X L) 1$ (100\%), tumor protein (TP)53 (54.75\%), Wilms' tumor (WT)1-exon7 (100\%), and WT1exon9 (43.77\%). Treatment with ATRA and ATO was discontinued, and diuretic detumescence and ventilatorassisted respiratory therapy were administered. A second BM smear showed 95.5\% abnormal promyelocytic granulocytes, with an occasional Auer body in the form of an Auer bundle; the rate of positive peroxidase staining was $100 \%$, suggesting no remission (Figure 1D and E). This indicated that the patient was completely resistant to ATRA and ATO, and the treatment was switched to the IA regimen (idarubicin $\left[10 \mathrm{mg} / \mathrm{m}^{2}\right.$ for 3 days] and cytarabine $[150 \mathrm{mg} /$ day for 7 days]) as induction therapy. The patient experienced severe pulmonary infection on day 7 after initiation of the regimen, and received antibiotics for 2 weeks until the infection was gradually controlled; however, WBC and platelet counts remained low, and a third BM smear showed $10.5 \%$ blasts. A HIAG chemotherapy regimen (homoharringtonine [2 $\mathrm{mg} /$ day] for 7 days, idarubicin [5 mg/day for 4 days], cytarabine [40 mg/day for 14 days], and granulocyte colony-stimulating factor [GCSF; $300 \mu \mathrm{g} /$ day until $\mathrm{WBC}$ count was $\left.>20 \times 10^{9} / 1\right]$ ) was administered as reinduction chemotherapy. A BM examination 
A

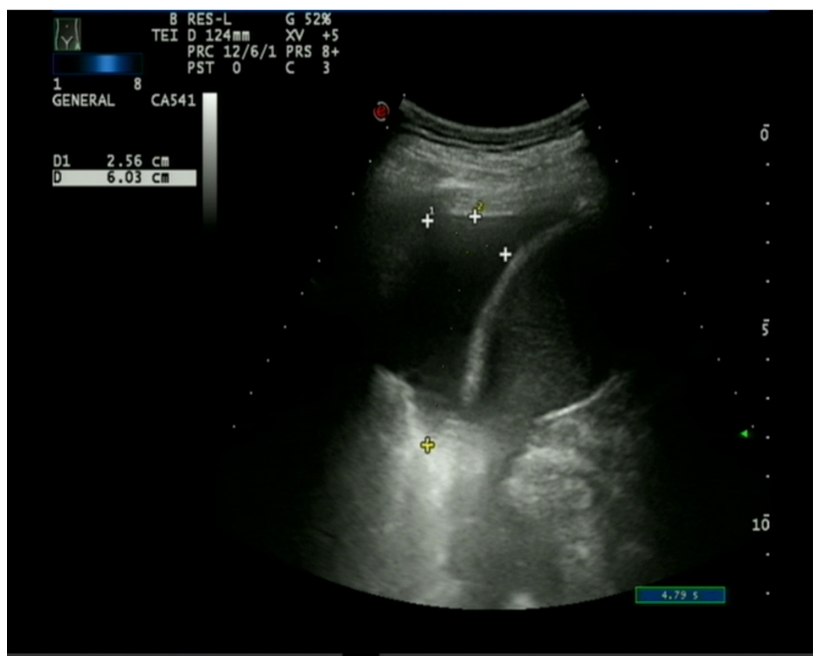

B

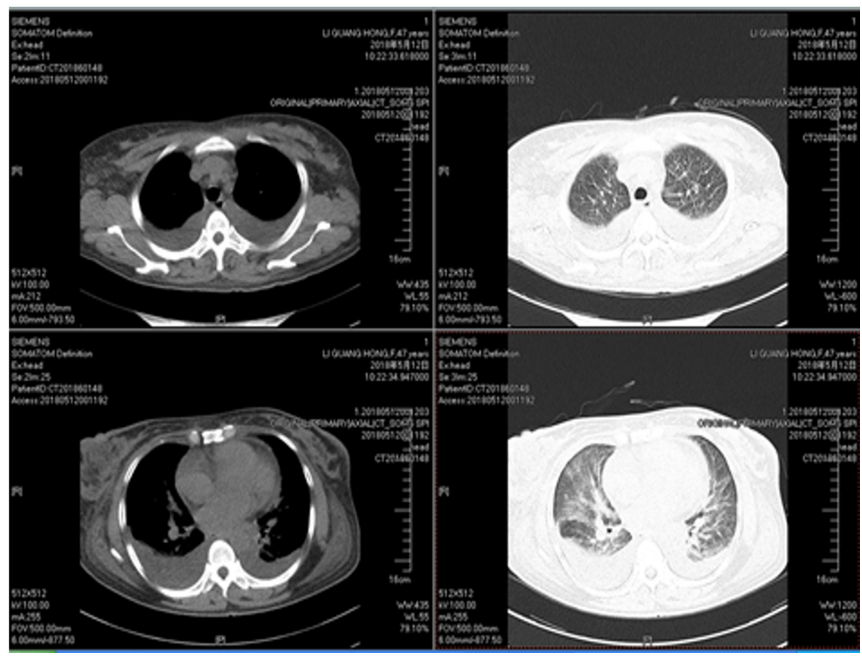

Figure 3 Chest computed tomography (CT) and color Doppler ultrasound of the patient. (A) Color Doppler ultrasound showing pleural effusion at a depth of $6.03 \mathrm{~cm}$. (B) Chest CT showing pleural effusion on both sides.

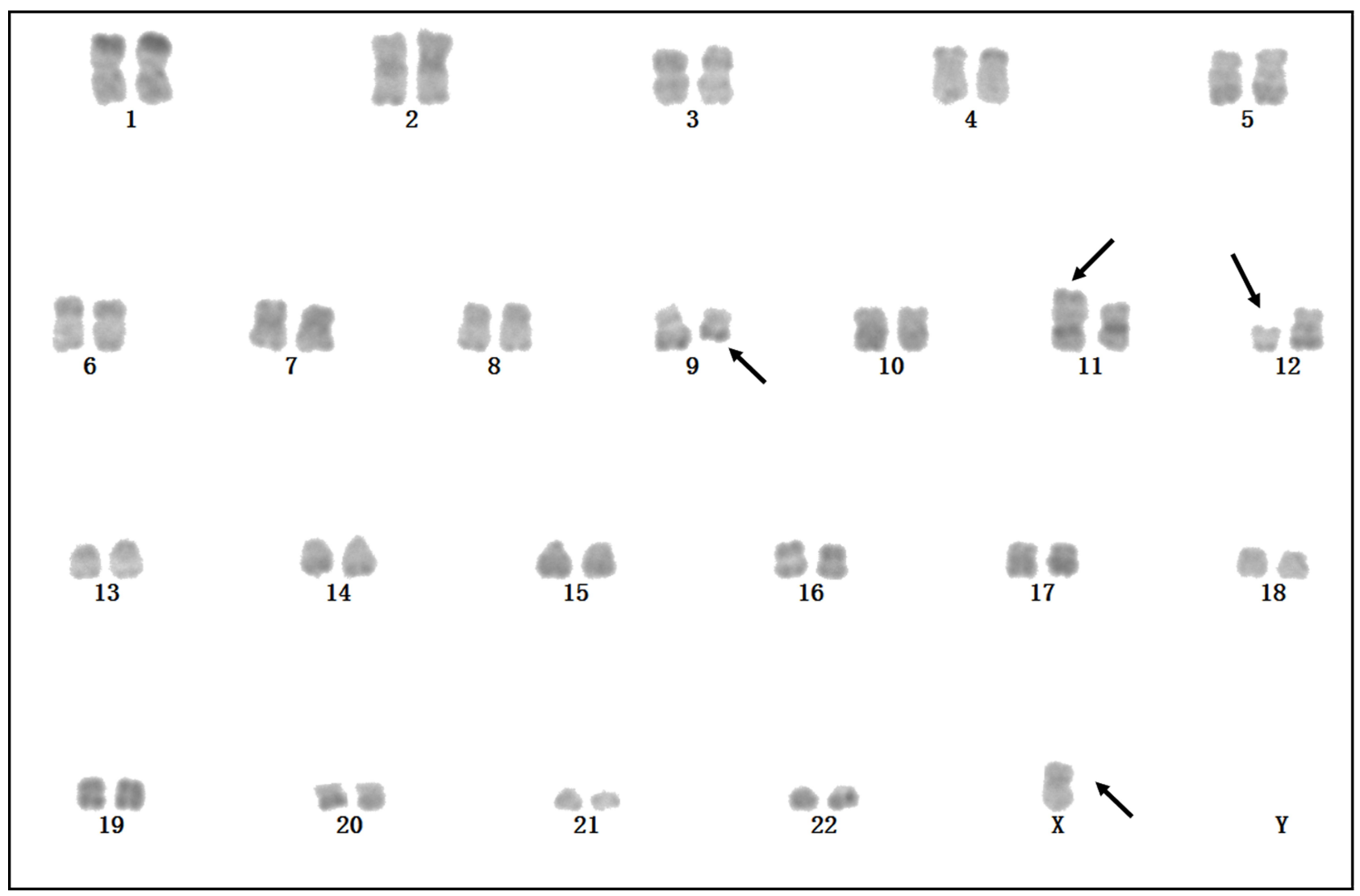

Figure 4 Karyotype of an AML patient with NUP98-RARG gene fusion. G-banding revealed a karyotype of 45, X, -X, del(9)(qI3q22), t(II; I2)(pI5; qI3); arrows indicate I Ip ,$+ 12 q^{-}$, and $9 q^{-}$. 
was performed to evaluate the response to HIAG; the smear showed $0.5 \%$ blasts, indicating that the patient had achieved complete remission (Figure 1F).

Consolidation therapy with the HIAG regimen was initiated on August 13, 2018. During the BM suppression period following chemotherapy, severe pulmonary infection, septic shock, metabolic acidosis, heart failure, and other serious complications occurred; the patient was admitted to the intensive care unit for emergency treatment, and recovered after 1 month. The patient refused to undergo allogeneic hematopoietic stem cell transplantation (allo-HSCT). Since September 19, 2018, the patient has received 2 cycles of half-dose $\mathrm{CAG}$ regimen (aclarubicin hydrochloride [20 mg/day for 4 days], cytarabine [40 mg/ day for 7 days], and GCSF [300 $\mu \mathrm{g}$ /day until WBC count was $\left.>20 \times 10^{9} / 1\right]$ ) and 2 cycles of the HA regimen (homoharringtonine [4 $\mathrm{mg} /$ day for 3 days] and cytarabine [150 mg/day for 7 days]) as consolidation chemotherapy. The minimal residual disease detected by flow cytometry was negative during each round of consolidation chemotherapy. The treatment timeline is shown in Figure 5. The patient remains alive and was leukemia-free at the last (24-month) follow-up.

\section{RT-PCR}

NUP98-RARG mRNA was reverse transcribed into cDNA using random hexamers, and PCR was performed using the following primers: NUP98 forward, 5'-GAG TAACCCAAGCCTCACAGC-3' and RARG reverse, 5'CCCATAGTGGTAGCCTGAGGAC-3'. A 200-bp product was specifically amplified from the patient's cDNA, representing the NUP98-RARG fusion product (Figure 6A).

\section{Cytogenetic Analysis and Fluorescence in situ Hybridization (FISH)}

A BM sample was processed after short-term culture (24 h) according to standard procedures. The chromosomes were stained by G-banding and the karyotype was determined according to International System for Human Cytogenetic Nomenclature recommendations. FISH was performed on 200 interphase cells using dual-color translocation probes (Figure 6B).

\section{Results and Discussion}

Nine patients harboring a NUP98-RARG, $P M L-R A R G$, CPSF6-RARG, or NPM1-RARG-NPM1 gene rearrangement have been described to date; their age, sex, and molecular and genetic characteristic are shown in Table 1. In 2011, Such et al first reported NUP98-RARG fusion in an AML patient with morphologic and immunophenotypic features resembling APL (Table 1, No.1), the patient discontinued ATRA treatment due to absence of a $P M L-R A R A$ fusion gene and was switched to a standard $3+7$ regimen with cytarabine and idarubicin, undergoing consolidation chemotherapy followed by autologous SCT. ${ }^{6}$ Patient 2 was a 64-year-old woman with $P M L-R A R G$ fusion (Table 1, No.2); her BM smears showed atypical hypergranular promyelocytes with Auer rods, and she received idarubicin and cytarabine chemotherapy after 9 days of ATRA treatment as well as 1 cycle of high-dose cytarabine consolidation chemotherapy followed by allo-HSCT. ${ }^{7}$ In addition to NUP98$R A R G$ fusion, CPSF6-RARG or NPM1-RARG-NPM1 fusion have been detected in AML patients who all showed the classic morphologic and immunophenotypic features of APL and were resistant to ATRA and ATO. The common

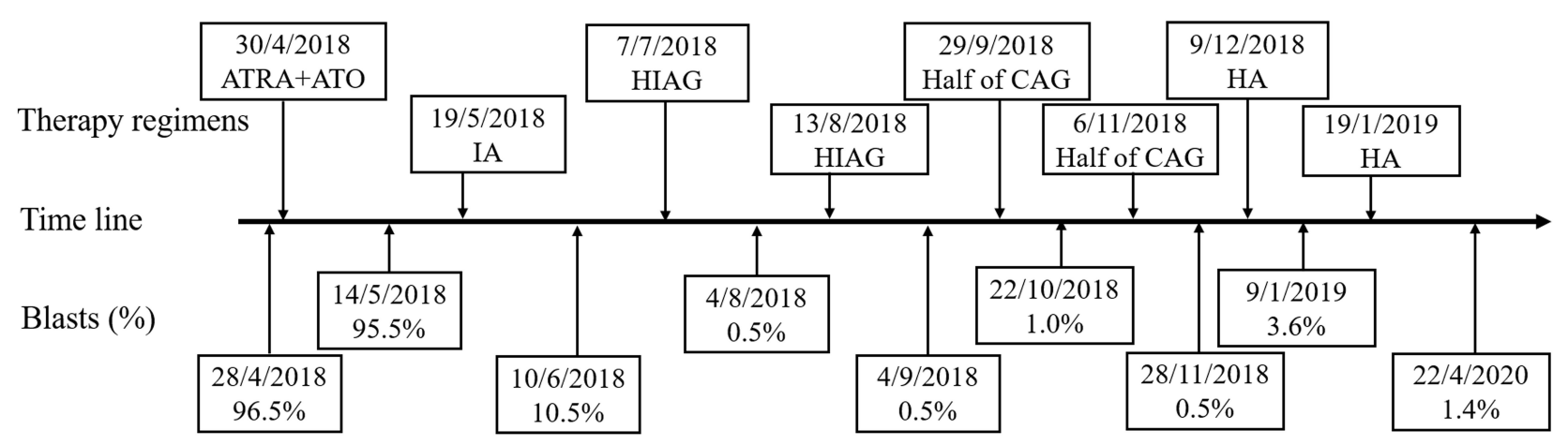

Figure 5 Timeline of patient's treatment. 
A

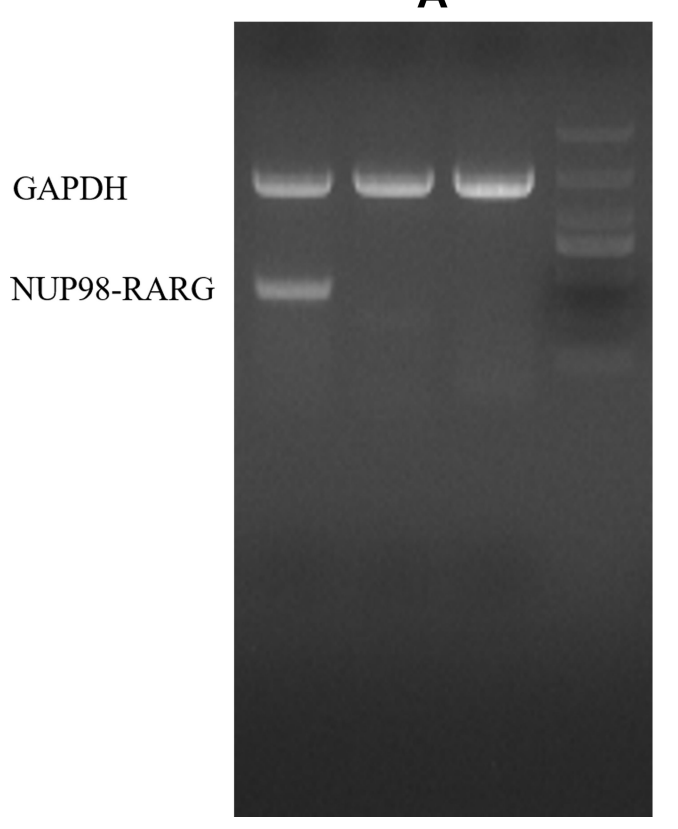

B

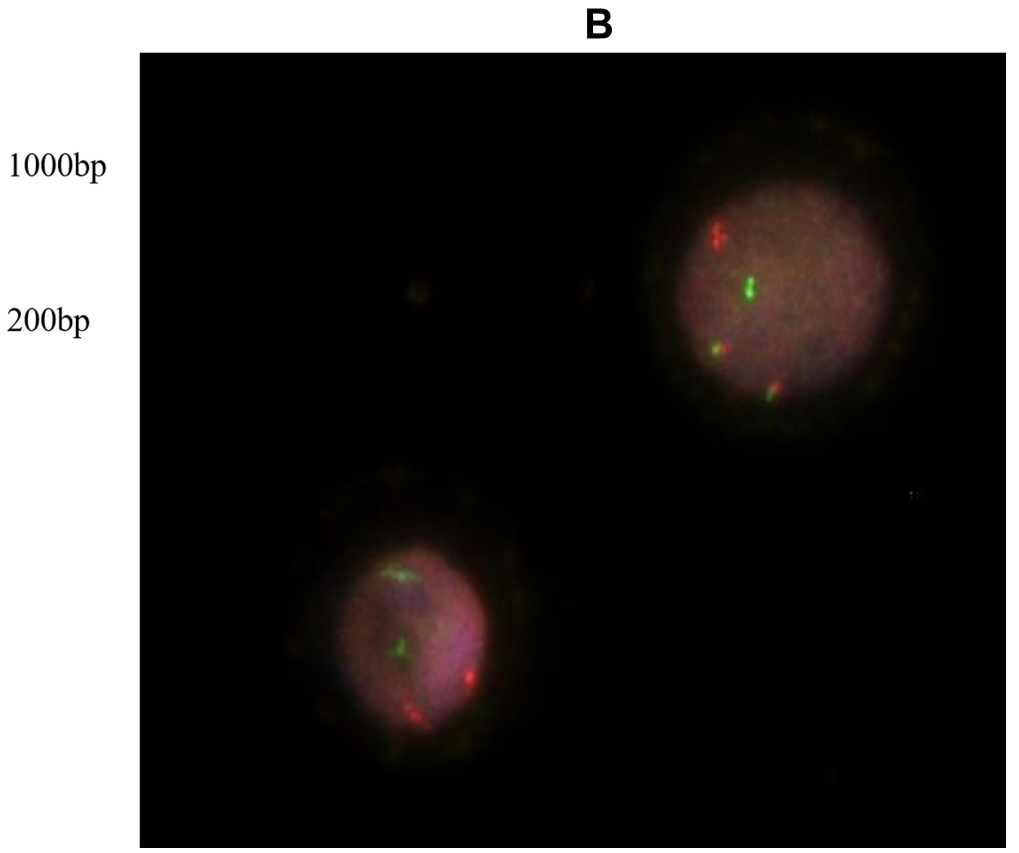

Figure 6 Detection of NUP98-RARG by RT-PCR and FISH. (A) NUP98-RARG mRNA was detected by RT-PCR analysis of leukemia cell total RNA derived from the AML patient; the product size was 200 bp. (B) FISH using dual color NUP98-RARG probes. The 2 orange signals show NUP98-RARG fusion, while green RARG and red NUP98 signals are present on intact chromosomes II and I2, respectively.

characteristic of these patients was the presence of $R A R G$ rearrangement. Some partner proteins can bind to different DNA-binding domains of $R A R G$ to form fusion proteins such as PML-RARG, NUP98-RARG, CPSF6-RARG, and NPM1-RARG-NPM1. NUP98 exon 12 was fused in frame to $R A R G$ exon 4, forming the NUP98-RARG fusion gene. ${ }^{5}$ However, the role of RARG in AML has yet to be elucidated.

The patient described herein was a 47-year-old female presenting with features of APL without a detectable $P M L-$ $R A R A$ fusion by RT-PCR. We detected the NUP98-RARG fusion gene in our patient by PCR using previously described primers (Figure 6A). The cytogenetic analysis revealed an intermediate-risk karyotype of 45, X, $-\mathrm{X}$, del (9)(q13q22), $\mathrm{t}$ $(11 ; 12)(\mathrm{p} 15 ; \mathrm{q} 13)$, and the NUP98-RARG fusion was also detected by FISH (Figure 6B). ATRA and ATO treatment was discontinued and switched to idarubicin and cytarabine. The patient experienced serious complications during the initial IA induction chemotherapy and subsequent low-dose regimen, and had poor compliance and refused allo-HSCT; we were also concerned about the risk of treatment-related death. Therefore, the patient received 6 cycles of low-dose

Table I Acute Myeloid Leukemia Resembling Acute Promyelocytic Leukemia with RARG Rearrangements in Literatures

\begin{tabular}{|c|c|c|c|c|c|}
\hline Case No. & $\begin{array}{l}\text { Sex/Age } \\
\text { (Years) }\end{array}$ & Cytogenetic Abnormal & $\begin{array}{l}\text { RARG } \\
\text { Rearrangements }\end{array}$ & $\begin{array}{l}\text { Survival } \\
\text { (Days) }\end{array}$ & Reference \\
\hline I & $M / 35$ & $\mathrm{t}(1 \mathrm{I} ; 12)(\mathrm{p}|5 ; \mathrm{q}| 3)$ & NUP98-RARG & 730 & Such, et al, $2011^{6}$ \\
\hline 2 & $\mathrm{~F} / 64$ & $\mathrm{t}(12 ; 15)(\mathrm{q} 13 ; \mathrm{q} 22)$ & PML-RARG & NA & $\mathrm{Ha}$, et al, $2017^{7}$ \\
\hline 3 & $M / 26$ & $45, X,-Y[10] /$ idem, add (6) (q?।3) [2] & RARG-CPSF6 & NA & Miller et al, $2018^{8}$ \\
\hline 4 & $\mathrm{~F} / 5 \mathrm{I}$ & $46, X X$, del $(12)(p \mid 2)[2] / 46, X X[18]$ & CPSF6-RARG & $>90$ & Liu et al, $2018^{9}$ \\
\hline 5 & $\mathrm{~F} / 48$ & $92, X X X X[2]$ & CPSF6-RARG & $<60$ & Liu et al, $2018^{9}$ \\
\hline 6 & $M / 38$ & $46, X Y[20]$ & CPSF6-RARG & 37 & Qin et al, $2018^{10}$ \\
\hline 7 & $M / 22$ & 46, XY, t (II;|2) (pI5; q|3) & NUP98-RARG & NA & Zhang et al, $2019^{11}$ \\
\hline 8 & $\mathrm{~F} / 45$ & $46, X X, t(I I ; \mid 2)(p|5 ; q| 3)[16]$ & NUP98-RARG & 35 & Luo et al, $2019^{12}$ \\
\hline 9 & $M / 69$ & $46, X Y$ & NPMI-RARG-NPMI & 240 & Chen et al, $2019^{13}$ \\
\hline 10 & $M / 55$ & $46, X Y$ & CPSF6-RARG & $>360$ & Zhang et al, $2020^{17}$ \\
\hline
\end{tabular}

Abbreviations: No., number; M, male; F, female; NA, not available. 
CAG and HA regimens as consolidation chemotherapy. At present, the patient is alive and in complete remission.

It was reported that NUP98 exon 12 is fused in-frame to $R A R G$ exon $4,{ }^{5}$ but the mechanism of oncogenic transformation mediated by $N U P 98-R A R G$ in AML is unknown. An in vitro study showed that the NUP98$R A R G$ fusion was extremely sensitive to ATRA treatment, implying that retinoid/rexinoid signaling plays an important role in AML and is a potential therapeutic target for patients harboring this chromosomal abnormality. ${ }^{14}$ However, another study examining relapsed primary blasts of an AML patient with NUP98-RARG rearrangement found that they were resistant to ATRA. ${ }^{15}$ Consistent with other case reports, we found that leukemia cells with NUP98-RARG fusion were completely resistant to both ATRA and ATO. Abnormalities in other genes such as WT1 and enhancer of zeste homolog 2 may confer ATRA resistance. ${ }^{10,16}$ Our patient also harbored 2 WT1 mutations and IDH2, TET2, ASXL1, or TP53 mutations. AML patients with $R A R G$ rearrangement can be treated with either idarubicin or homoharringtonine and cytarabine as the induction chemotherapy regimen, ${ }^{6,11,17}$ while allo-HSCT is recommended for post-remission treatment.

In summary, previous reports and findings from our case demonstrate that NUP98-RARG rearrangement defines a novel subtype of AML with morphologic and immunologic characteristics similar to APL but showing resistance to ATRA and ATO treatment. Further studies are needed to clarify the mechanism by which NUP98 $R A R G$ fusion promotes leukemogenesis and confers ATRA resistance in this subset of AML patients.

\section{Patient Statement}

Written, informed consent for publication of the case details was obtained from the patient.

\section{Abbreviations}

allo-HSCT, allogeneic hematopoietic stem cell transplantation; AML, acute myeloid leukemia; APL, acute promyelocytic leukemia; ASXL1, additional sex combs-like 1; ATO, arsenic trioxide; ATRA, all-trans retinoic acid; $\mathrm{BM}$, bone marrow; CD, cluster of differentiation; FISH, fluorescence in situ hybridization; GCSF, granulocyte colony-stimulating factor; $\mathrm{Hg}$, hemoglobin; IDH2, isocitrate dehydrogenase 2; NUP98, nucleoporin 98; RARA, retinoic acid receptor alpha; RARG, retinoic acid receptor gamma; RT-PCR, reverse transcription-polymerase chain reaction;
TET2, ten-eleven translocation 2; TP53, tumor protein 53; WBC, white blood cell; WT1, Wilms' tumor 1.

\section{Funding}

This work was supported by research grants from the Science and Technology Fund of Huai' an City (no. HAB201810) and Science and Technology Fund of Jiangsu Commission of Health (nos. H2018085 and H2019082).

\section{Disclosure}

The authors report no conflicts of interest in this work. Cofirst authors: Shandong Tao, Lixiao Song.

\section{References}

1. Short NJ, Rytting ME, Cortes JE. Acute myeloid leukaemia. Lancet. 2018;392(10147):593-606. doi:10.1016/S0140-6736(18)31041-9

2. Szotkowski T, Faber E, Hubacek J, et al. Acute promyelocytic leukemia successfully treated also in elderly patients with significant comorbidities: a 20-year single-center experience. Neoplasma. 2015;62(01):146-151. doi:10.4149/neo_2015_019

3. Lo-Coco F, Avvisati G, Vignetti M, et al. Retinoic acid and arsenic trioxide for acute promyelocytic leukemia. $N$ Engl J Med. 2013;369 (2):111-121. doi:10.1056/NEJMoa1300874

4. Sanz MA, Fenaux P, Tallman MS, et al. Management of acute promyelocytic leukemia: updated recommendations from an expert panel of the European leukemianet. Blood. 2019;133(15):1630-1643. doi:10.1182/blood-2019-01-894980

5. Conserva MR, Redavid I, Anelli L, et al. RARG gene dysregulation in acute myeloid leukemia. Front Mol Biosci. 2019;6:114.

6. Such E, Cervera J, Valencia A, et al. A novel NUP98/RARG gene fusion in acute myeloid leukemia resembling acute promyelocytic leukemia. Blood. 2011;117(1):242-245. doi:10.1182/blood-2010-06-291658

7. Ha JS, Do YR, Ki CS, et al. Identification of a novel PML-RARG fusion in acute promyelocytic leukemia. Leukemia. 2017;31 (9):1992-1995. doi:10.1038/leu.2017.167

8. Miller CA, Tricarico C, Skidmore ZL, et al. A case of acute myeloid leukemia with promyelocytic features characterized by expression of a novel RARG-CPSF6 fusion. Blood Adv. 2018;2(11):1295-1299. doi:10.1182/bloodadvances.2017014183

9. Liu T, Wen L, Yuan $\mathrm{H}$, et al. Identification of novel recurrent CPSF6-RARG fusions in acute myeloid leukemia resembling acute promyelocytic leukemia. Blood. 2018;131(16):1870-1873. doi:10. 1182/blood-2017-11-818716

10. Qin YZ, Huang XJ, Zhu HH. Identification of a novel CPSF6-RARG fusion transcript in acute myeloid leukemia resembling acute promyelocytic leukemia. Leukemia. 2018;32(10):2285-2287. doi:10.1038/s41375-018-0095-z

11. Zhang X, Li F, Wang J, et al. RAR $\gamma$-rearrangements resemble acute promyelocytic leukemia and benefit from $3+7$ regimen. Leuk Lymphoma. 2019;60(7):1831-1834. doi:10.1080/10428194.2018.15 53302

12. Luo H, Zhang S, Li K, et al. A novel entity of acute myeloid leukaemia with recurrent RARG-rearrangement resembling acute promyelocytic leukaemia. Leuk Res. 2019;77:14-16. doi:10.1016/j. leukres.2018.12.009

13. Chen X, Wang F, Zhang Y, et al. A novel NPM1-RARG-NPM1 chimeric fusion in acute myeloid leukaemia resembling acute promyelocytic leukaemia but resistant to all-trans retinoic acid and arsenic trioxide. $B r J$ Cancer. 2019;120(11):1023-1025. doi:10. 1038/s41416-019-0456-z 
14. Qiu JJ, Zeisig BB, Li S, et al. Critical role of retinoid/rexinoid signaling in mediating transformation and therapeutic response of NUP98-RARG leukemia. Leukemia. 2015;29(5):1153-1162. doi:10. 1038/leu.2014.334

15. Such E, Cordón L, Sempere A, et al. In vitro all-trans retinoic acid sensitivity of acute myeloid leukemia blasts with NUP98/RARG fusion gene. Ann Hematol. 2014;93(11):1931-1933. doi:10.1007/ s00277-014-2073-5
16. Coccaro N, Zagaria A, Orsini P, et al. RARA and RARG gene downregulation associated with EZH2 mutation in acute promyelocytic-like morphology leukemia. Hum Pathol. 2018;80:82-86. doi:10.1016/j. humpath.2018.02.023

17. Zhang Z, Jiang M, Borthakur G, et al. Acute myeloid leukemia with a novel CPSF6-RARG variant is sensitive to homoharringtonine and cytarabine chemotherapy. Am J Hematol. 2020;95(2):E48-E51. doi:10.1002/ajh.25689

\section{Publish your work in this journal}

OncoTargets and Therapy is an international, peer-reviewed, open access journal focusing on the pathological basis of all cancers, potential targets for therapy and treatment protocols employed to improve the management of cancer patients. The journal also focuses on the impact of management programs and new therapeutic

Submit your manuscript here: https://www.dovepress.com/oncotargets-and-therapy-journal agents and protocols on patient perspectives such as quality of life, adherence and satisfaction. The manuscript management system is completely online and includes a very quick and fair peer-review system, which is all easy to use. Visit http://www.dovepress.com/ testimonials.php to read real quotes from published authors. 